\title{
The Clinical Effect and Meta-analysis of Mesenchymal Stem Cells for Periodontal Tissue Regeneration
}

Nannan Han ${ }^{1}$, Yingying $\mathrm{Su}^{2}$, Lijia Guo ${ }^{3}$, Lu Jia ${ }^{1}$, Juan Du ${ }^{1}$, Hao Wang ${ }^{2}$, Yi Liu $^{1 *}$

${ }^{1}$ Laboratory of Tissue Regeneration and Immunology and Department of Periodontics, Beijing Key Laboratory of Tooth Regeneration and Function Reconstruction, School of Stomatology, Capital Medical University, P.R. China

${ }^{2}$ Department of Stomatology, Beijing Tiantan Hospital, Capital Medical University, P.R. China

${ }^{3}$ Department of Orthodontics School of Stomatology, Capital Medical University, P.R. China

"Corresponding author: Yi Liu, Laboratory of Tissue Regeneration and Immunology and Department of Periodontics, Beijing Key Laboratory of Tooth Regeneration and Function Reconstruction, School of Stomatology Capital Medical University, Tian Tan Xi Li No.4, Beijing 100050, P.R. China, Tel: 86-10-57099450; Fax: 86-10-57099450; E-mail: lililiuyi@163.com

Received date: August 04, 2018; Accepted date: August 20, 2018; Published date: August 22, 2018

Copyright: () 2018 Han N. This is an open-access article distributed under the terms of the Creative Commons Attribution License, which permits unrestricted use, distribution, and reproduction in any medium, provided the original author and source are credited.

\begin{abstract}
Objective: To probe the potency and safety of transplantation of Mesenchymal Stem Cells (MSCs) in periodontal tissue regeneration.

Methods: The following electronic data bases were used to retrieve: Cochrane Library, PubMed, Ovid, CBM, and CNKI Data from its incipiency to December 2017. Inclusion criteria were clinical studies in humans, on the application of MSCs in periodontal tissue regeneration. Meta-analysis was implemented only for experimental studies (Randomized Controlled Trials (RCTs) and Controlled Trials (CTs)) concerning periodontal tissue regeneration. The two authors screened the literature according to inclusion and exclusion criteria, extracted data, estimated the risk of bias. The meta-analysis was applied using Review Manager, version 5.1.
\end{abstract}

Results: 4 studies and 109 teeth were included in this meta-analysis. The results demonstrated that the use of MSCs group was preferable to the control group with statistical significance in the PD (SMD $=0.79,95 \% \mathrm{Cl}[0.39$, 1.18], $\left.I^{2}=0 \%, P<0.0001\right)$ and $C A L\left(M D=0.54,95 \% C l[0.16,0.92], I^{2}=78 \%, P=0.005\right)$. No serious adverse events were reported.

Conclusion: It is suggested that the MSCs-based therapy for periodontal tissue regeneration is effective. However, more high-quality evidences are still required for further inquiry to promote clinical utilization of MSCs.

Keywords: Mesenchymal stem cells transplantation; Periodontal tissue regeneration; Metanalysis

Abbreviations: MSCs: Mesenchymal Stem Cells; RCTs: Randomized Controlled Trials; GTR: Guided Tissue Regeneration; PD: Probing Depth; CAL: Clinical Attachment Level; GR: Gingival Recession; PDL: Periodontal Ligament; SD: Standard Deviation; SE: Standard Error; SMD: Standardized Mean Difference; CIs: Confidence Intervals

\section{Introduction}

Periodontitis is a chronic multifactorial disease of the periodontal tissues and is characterized by the destruction of the periodontium, including the loss of cementum, Periodontal Ligament (PDL), and alveolar bone, eventually leading to periodontal defects [1]. It is particularly known that chronic periodontitis is caused by bacterial infection and host immune response [2,3]. The terminal objective of periodontal treatment is to restore and reform the demolished periodontal tissues, which consists of gingival connective tissue, PDL, cementum and alveolar bone. At present, periodontitis treatment includes conventional approaches such as oral hygiene instructions, scaling and root planning. However, these approaches could not obtain desired the regeneration of damaged periodontal tissues stays a clinical challenge [4]. New periodontitis treatments contain the introduction of bone grafts, Guided Tissue Regeneration (GTR), gene therapy, and a variety of growth factor-mediated treatments. However, to date these tactics fail to accurately achieve the thorough new periodontal tissues impaired by severe periodontitis [5-7].

At present, MSCs-based periodontal tissue regeneration is identified as a promising way of periodontitis therapies, especially periodontal tissue engineering as a treatment for periodontitis. MSCs are reliable seed cells for tissue regeneration owing to their multilineage differentiation and self-renewal characteristics [8-10]. In fact, effective preclinical outcomes have been acquired in an extensive variety of in vitro and in vivo models [11-24]. On the basis of these preclinical studies, recent studies have been concentrated on stem cells transplantation therapies to treat periodontal tissue defects in the clinic. MSCs can be injected directly into the intrabony defects as a way of cell suspension or applied with biomaterial scaffolds. Although several publications in clinical studies, the effectiveness of MSCmediated methods for periodontal tissue regeneration remains inconsistent. Some studies showed that MSC-based treatment methods had a positive impact on periodontal tissue regeneration in comparison with control groups [25-28], while others revealed that there were no significant statistical differences between experimental groups with MSCs and control groups without MSCs [29-30]. Therefore, these paradoxical results have posed doubt about the safety 
and capacity of MSC-mediated ways of the improvement of periodontal tissue regeneration.

Thus, the aim of this study was to implement a systematical review and a meta-analysis with regard to MSC-based way of periodontal tissue regeneration in clinical studies to get more clinical evidence on their effectiveness.

\section{Materials and Methods}

\section{Study design}

The methods of systematic review were pre-specified and recorded in the protocol executed by the review authors. The type of studies selection, appraisal of risk of bias, data extraction, and report quality were operated by two normative reviewers ( $\mathrm{HNN}$ and JL) in duplicate. The dissents between them were settled by argument and the unresolved question was communicated to a third author to achieve consensus.

\section{Inclusion and exclusion criteria}

Studies in conformity with the inclusion criteria are as follows: 1) most important type of study designs to resolve the center issues were RCTs and CTs; 2) participants who were diagnosed as periodontal disease by clinical and radiological estimation without restriction in age, race, gender, and social and economic status; 3 ) participants were in good general health without any symbol of systematic disease; 4) The clinical outcomes of management from baseline to follow-up time points (or before/after therapy) should include Probing Depth (PD), Clinical Attachment Level (CAL) and Gingival Recession (GR); 5) all participants included in this study received standard initial periodontal therapy. Exclusion criteria were studies where participants were smoking within the last 6 months before surgery and pregnancy. Literature reviews, meta-analysis, repeated studies, and experimental model researches were also excluded.

\section{Search strategy}

Systematic searches were performed in Cochrane Library, PubMed, Ovid, CBM, CNKI, Data from its inception to December 2017, to collect RCTs and CTs of stem cell transplantation for periodontal tisuue regeneration. Published languages involve English and Chinese. Medical Subject Headings (MeSH) and free words such as "stem cell," "stem cells," "cells," "mesenchymal stem cells," "transplantation," "implantation," "cells implantation," "mesenchymal stem cell (MSC) transplantation," "cell-based therapy," "periodontitis," "periodontal diseases," "periodontal intrabony defects," "periodontal tissue regeneration," "periodontal tissue restoration," "guided tissue regeneration," and "periodontium repair." The search methods were combined with the Cochrane Highly Sensitive Search Strategy to distinguish randomized control trials.

The titles and abstracts were first filtrated to obtain any research that matched the requirements, and full texts were attained for further consideration.

\section{Risk of bias assessment}

Assessed the risk of bias were estimated by two authors independently according to the inclusion and exclusion criteria. The Cochrane Collaboration tool was used to assess the risk of bias including the following terms: (1) sequence generation, (2) allocation concealment, (3) blinding of participants, (4) blinding of outcome assessors, (5) incomplete outcome, (6) selective data reporting, and (7) other biases [15]. The risk of bias of each field was ranged as follows: A, low risk; $\mathrm{B}$, unclear risk; and $\mathrm{C}$, high risk. In addition, for the included studies the risk of bias of was further categorized as one of the following classifications: "a low risk of bias" if the 7 fields were assessed as A; "an unclear risk of bias" if one or more fields were categorized as $\mathrm{B}$; and "a high risk of bias" if one or more fields were categorized as C. For each outcome, the risk of bias of depended on the indispensable fields. For the objective appraisal, the fundamental fields were fields 1 , $2,5,6$, and 7 , and for the subjective evaluation, the master fields were fields $1,2,3,5,6$, and 7 .

\section{Data extraction}

Extracted data in the light of the inclusion and exclusion criteria was by two authors independently. A data extraction table was designed and extracted data from the included studies. The following terms were extracted: including authors, year of paper publication, patients' average age, gender, treatment group/control group, number of teeth, follow-up, and outcome appraisals, mean value, SD (Standard Deviation) or SE (Standard Error). If the outcomes were evaluated for $>1$ time points, only included the last time of assessment was included.

\section{Data analysis/statistical analysis}

Reviewer Manager 5.1 was chosen for the quantitative data of mean difference (MD) or Standardized Mean Difference (SMD) and 95\% Confidence Intervals (CIs) to meta-analysis. $\mathrm{I}^{2}$ was used to evaluate heterogeneity. If no remarkable heterogeneity or low heterogeneity between researches was observed, the fixed effects model was utilized. If the significant statistical heterogeneity between researches was detected, the reasons for heterogeneity would be analyzed. Subgroup analysis and random-effects model would be used instead of fixedeffects model. Finally, to assess the publication bias, the funnel plot analysis was performed.

\section{Results}

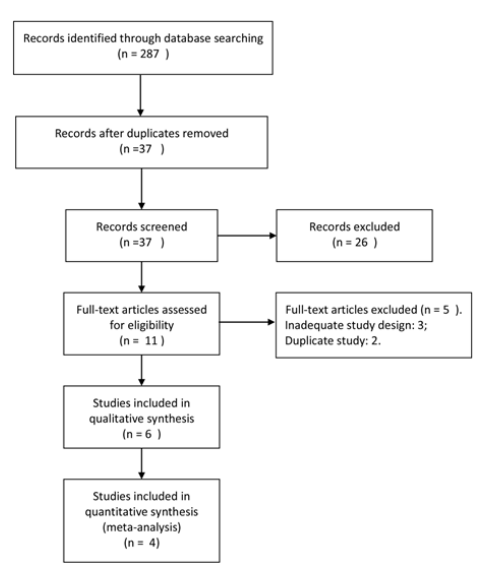

Figure 1: Flow of the study inclusion of systemic review. 
Citation: Han N, Su Y, Guo L, Jia L, Du J, et al. (2018) The Clinical Effect and Meta-analysis of Mesenchymal Stem Cells for Periodontal Tissue Regeneration. Dentistry 8: 508. doi:10.4172/2161-1122.1000508

Page 3 of 6

\section{Study characteristics}

A total of 287 papers were screened by electronic retrievals. After identifying based on the titles and abstracts, 11 studies were taken into consideration to be qualified, and full texts of them were recovered. Finally, 4 studies included and 5 studies were excluded (Figure 1).
All the included studies were parallel RCTs with 109 teeth diagnosed as periodontal intrabony defects. Other two studies reported as clinical studies. The follow-up period changed from 3 to 12 months (Table 1).

\begin{tabular}{|c|c|c|c|c|c|c|c|}
\hline \multicolumn{8}{|l|}{ Intervention } \\
\hline Investigator & $\begin{array}{l}\text { Gender } \\
\text { (M/F) }\end{array}$ & Age & $\begin{array}{l}\text { Numbers } \\
\text { teeth (I/C) }\end{array}$ & Intervention Group & Control Group & Follow-up (mo) & Outcome \\
\hline Chen 2016 & Aug-30 & $30.04 \pm 7.9$ & $20 / 21$ & dental MSCs + Bio-oss & Bio-oss & $3,6,12$ & PD, CAL \\
\hline Kushal 2017 & $x$ & 35 & $16 / 14$ & $\begin{array}{l}\text { Umbilical Stem cells }+ \\
\text { PLA/PGA }\end{array}$ & PLA/PGA & 6 & PD, CAL, GR \\
\hline Dhote 2015 & $x$ & $32.62 \pm 6.99$ & 12-Dec & $\begin{array}{l}\text { Umbilical MSCs + rh- } \\
\text { PDGF-BB }\end{array}$ & OFD & 6 & PD, CAL, \\
\hline Riccardo 2009 & 01-Jun & $30.29 \pm 5.87$ & 07-Jul & dental MSCs + Scaffold & Scaffold & 12 & PD \\
\hline
\end{tabular}

Table 1: Characteristics of included studies.

\section{Quality assessment and bias risk}

Most studies had an unclear risk of bias (Table 2).

\begin{tabular}{|c|c|c|c|c|c|c|c|}
\hline \multicolumn{8}{|l|}{ Domain } \\
\hline Investigator & 1 & 2 & 3 & 4 & 5 & 6 & 7 \\
\hline Chen 2016 & A (random number) & A & A & A & A & A & A \\
\hline Kushal 2017 & A (coin flip) & B & B & B & B & A & A \\
\hline Dhote 2015 & B & B & B & B & A & A & A \\
\hline Riccardo 2009 & B & B & B & B & A & A & A \\
\hline
\end{tabular}

Table 2: Risk of bias assessment of included studies.

\section{Meta-analysis and effect evaluation}

A meta-analysis of 4 studies was conducted to give a quantitative assessment of the SMD of PD between the experimental and control group. The four studies reported changes of PD reduction [27-30]. Three studies pointed out CAL gains enhancement [28-30], however, GR reduction was no significant change. The outcomes of metaanalysis were as below: PD (SMD $=0.79,95 \%$ CI $[0.39,1.18], \mathrm{I}^{2}=0 \%$, $\mathrm{P}<0.0001$, (Figure 2), CAL ( $\mathrm{MD}=0.54,95 \%$ CI $[0.16,0.92], \mathrm{I}^{2}=78 \%$, $\mathrm{P}=0.005$, (Figure 3), GR ( $\mathrm{MD}=-0.19,95 \%$ CI $[-0.5,0.12], \mathrm{I}^{2}=77 \%$, $\mathrm{P}=0.23$ (Figure 4). The outcomes of $\mathrm{PD}$ and $\mathrm{CAL}$ after the combination were beneficial to the stem cell implantation group. The differences were statistically significant.

$\mathrm{I}^{2}$ index was utilized to measure the heterogeneity. The formal experiment testified that GR and CAL were the existence of extensive heterogeneity (GR: $\mathrm{I}^{2}=77 \%, \mathrm{P}=0.23$; CAL: $\mathrm{I}^{2}=78 \%, \mathrm{P}=0.005$ ). In order to analyse the substantial heterogeneity was detected, subgroup analysis was used to further estimate the clinical effectiveness on the basis of two clinical variables, and we concentrated on three clinical variables: type of MSCs interventions, type of control group interventions, and follow-up time period. To analyse each clinical variable by interplaying with GR or CAL changes which were observed coincidentally, including assessment of subgroup outcomes and heterogeneity analysis (Tables 3 and 4). Therefore, when the follow-up periodtype of control group with/without scaffold, and type of MSC were analyzed, GR and CAL were improved respectively. However, the heterogeneity was still existed.

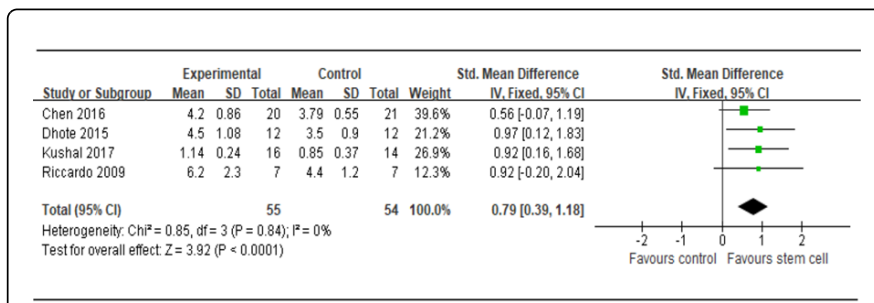

Figure 2: Meta-analysis of MSCs treatment versus no MSCs for reduction.

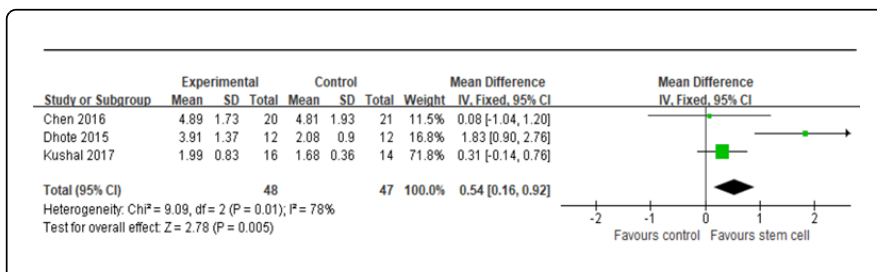

Figure 3: Meta-analysis of MSCs treatment versus no MSCs for improved CAL gains. 
Citation: Han N, Su Y, Guo L, Jia L, Du J, et al. (2018) The Clinical Effect and Meta-analysis of Mesenchymal Stem Cells for Periodontal Tissue

\begin{tabular}{|c|c|c|c|c|c|c|c|c|c|}
\hline \multirow[b]{2}{*}{ Study or Subgroup } & \multicolumn{3}{|c|}{ Experimental } & \multicolumn{3}{|c|}{ Control } & \multicolumn{2}{|c|}{ Mean Difference } & \multirow{2}{*}{$\begin{array}{l}\text { Mean Difference } \\
\text { IV. Fixed, } 95 \% \mathrm{Cl}\end{array}$} \\
\hline & Mean & SD & Total & Mean & SD & Total & Weight & NV. Fixed, $95 \% \mathrm{Cl}$ & \\
\hline Chen 2016 & 1.28 & 0.82 & 20 & 1.54 & 0.96 & 21 & $32.6 \%$ & $-0.26[-0.81,0.29]$ & \\
\hline Dhote 2015 & 0.58 & 0.79 & 12 & 1.4 & 0.66 & 12 & $28.6 \%$ & $-0.82[-1.40,-0.24]$ & $\rightarrow-$ \\
\hline Kushal 2017 & 1.57 & 0.89 & 16 & 1.24 & 0.47 & 14 & $38.7 \%$ & $0.33[-0.17,0.83]$ & \\
\hline Total $(95 \% \mathrm{Cl})$ & & & 48 & & & 47 & $100.0 \%$ & $-0.19[-0.50,0.12]$ & \\
\hline $\begin{array}{l}\text { Heterogeneity. } \mathrm{Ch}^{2}= \\
\text { Test for onerall effect }\end{array}$ & $\begin{array}{l}8.70, \mathrm{df}= \\
Z=1.21\end{array}$ & $\begin{array}{l}=2(P \\
(P=0\end{array}$ & $\begin{array}{l}=0.01) \\
23)\end{array}$ & $P^{2}=77$ & & & & & $\begin{array}{cccc}2 & -1 & 0 & 1 \\
\text { rs control } & \text { Favours }\end{array}$ \\
\hline
\end{tabular}

Figure 4: Meta-analysis of MSCs treatment versus no MSCs for GR reduction.

\begin{tabular}{|c|c|c|}
\hline Subgroup & Mean difference $(95 \% \mathrm{Cl})$ & $\mathrm{I}^{2}(\%)$ \\
\hline \multicolumn{3}{|l|}{ Follow-up period } \\
\hline$<6$ months & $-0.26(-0.81,0.29)$ & - \\
\hline$\geq 6$ months & $-0.19(-0.75,0.37)$ & 86 \\
\hline \multicolumn{3}{|c|}{ Type of control group } \\
\hline Scaffold & $0.06(-0.31,0.43)$ & 59 \\
\hline Non-scaffold & $-0.82(-1.4,-0.24)$ & - \\
\hline \multicolumn{3}{|l|}{ Cell type } \\
\hline Dental MSC & $-0.26(-0.81,0.29)$ & - \\
\hline Non-dental MSC & $-0.19(-0.75,0.37)$ & 86 \\
\hline
\end{tabular}

Table 3: The heterogeneity of GR was studied by subgroup analysis.

\begin{tabular}{|l|l|l|}
\hline Subgroup & Mean difference $(95 \% \mathrm{Cl})$ & I2 (\%) \\
\hline Follow-up period & $0.04(-0.57,0.66)$ & - \\
\hline$<6$ months & $0.87(0.29,1.44)$ & 68 \\
\hline$\geq 6$ months & $0.28(-0.14,0.69)$ & 0 \\
\hline Type of control group & $1.52(0.60,2.45)$ & - \\
\hline Scaffold & & \\
\hline Non-scaffold & $0.04(-0.57,0.66)$ & - \\
\hline Cell type & $0.87(0.29,1.44)$ & 68 \\
\hline Dental MSC &
\end{tabular}

Table 4: The heterogeneity of CAL gains was studied by subgroup analysis.

\section{Discussion}

MSCs appear to be a promising method in the context of tissue engineering and cell-based therapy. MSCs have also been

demonstrated to form new cementum, PDL and alveolar bone in vivo after transplantation into periodontal lesions in animal models [11-25], suggesting that MSCs may be useful seed cells for periodontal tissue regeneration. Tassi et al. estimated 22 researches on preclinical experimental model of MSCs treatment through a systematic review and demonstrated that MSC-mediated treatment had promoted the animals' periodontal regeneration and diminished the inflammation of periodontal lesions [31]. Yan et al. surveyed the influence of preclinical mesenchymal stromal cells transplantation on animal models of periodontitis and demonstrated that MSC had favorable therapeutic effect, and the results were related to the type of MSC, approach of transplantation, newly bone formation, cementum and PDL [32]. Therefore, according to these triumphant results of preclinical animal experimental studies, the clinical application of MSCs for periodontal tissue regeneration in humans has begun. Feng et al. used the local adhibition of autologous Periodontal Ligament Stem Cells (PDLSCs) or gingival stem cells to treat patients with periodontitis in the previous study, the results showed that neither obvious adverse effects nor an enhanced significantly any autoantibodies were detected; however, there were only three case reports of long-term observation [13]. To transfer MSC-mediated periodontal therapeutic approach from preclinical experimental research and case report into clinical application, Chen et al. evaluated the safety and ability of utilizing autologous PDLSCs as an auxiliary to graft materials in GTR to treat periodontal intrabony lesions caused by periodontitis, the results revealed that a compelling raised in the height of alveolar bone (decrease in the bone lesion depth) as time gone on, suggesting that the application of autologous PDLSCs to treat periodontal bone lesions is effective and does not induce remarkable adverse reactions [29]. All those studies confirmed that the MSC-mediated periodontal tissue regeneration has a promising utilization in the future dental clinic.

The objective of this research was to systematically assess the current evidence for the validity of MSC-mediated treatments for periodontal tissue regeneration in clinical applications. In this study, a systematic review and meta-analysis of RCT studies on stem cell implantation for periodontal defects were conducted to analyze the possible publication bias and to explore the effects of cell-based methods on periodontal tissue regeneration, as shown by the positive impact of detected outcomes on new cementum, PDL, and alveolar bone formation. A total of 4 studies engaged 109 teeth with intrabony defects, and the patients with periodontal disease must have met WHO diagnostic criteria of periodontitis. Aggressive periodontitis was ruled 
out in these patients by CT or X-ray. Each group must have more than 5 patients. The type of interventions comprised dental tissue-derived MSCs, and non-dental tissue-derived MSCs. Finally, a meta-analysis was used to analyse 4 studies and 55 teeth treatment interventions; the clinical outcomes of therapeutic approaches from baseline to follow-up time points involved $\mathrm{PD}, \mathrm{CAL}$, and GR which all improved to some extent, with no obvious adverse reactions. The present systematic review implicated that the outcomes of $\mathrm{PD}$ and CAL after the consolidation were propitious to MSCs transplantation group, and there are significant differences in statistics. However, the formal test confirmed that GR and CAL were the exsitence of substantial heterogeneity (GR: $\mathrm{I}^{2}=77 \%, \mathrm{P}=0.23$; CAL: $\mathrm{I}^{2}=78 \%, \mathrm{P}=0.005$ ). To account for the expected heterogeneity, we used 3 clinical variables (type of MSCs interventions, type of control group interventions, and follow-up period) to interplay with 2 outcome variables (CAL and GR) and found that the GR of patients who received non-dental tissuederived stem cells transplantation were superior to those who applied dental tissue-derived stem cells. Some previous studies compared the reproductive capacity of dental tissue-derived stem cells with BMSCs (bone marrow stem cells), and the results revealed that dental tissuederived stem cells were the more eligible cell population for periodontal tissue regeneration, as remarkable more well-oriented PDL fibers, newly formation of cementum and alveolar bone were discovered on transplantation of dental tissue-derived stem cells [24, 33]. However, in the current study the results of these subgroup analyses showed that there were opposite results. In addition, when the follow-up time was analyzed, the outcome of CAL was improved, however, the analysis of these subgroups did not significantly decrease the heterogeneity. The main cause is that a lot of studies had flaws in the aspects of blindness, the integrity of data, trial designs, and the number of experiments in subgroups was less, and there was still a certain bias in the process of evaluation.

\section{Conclusion}

In conclusion, the current systematic review and meta-analysis indicate that MSC-based therapies have a beneficial influence on periodontal regeneration compared to control groups. However, as a consequence of less clinical trials of MSCs transplantation treatment, the less periodicity of participants, and possible the risk of bias problems in clinical trial designs, the occurrence of possible bias was still existed. As a result, further higher quality and more experimental data researches are still required to prove the safety and effectiveness of MSCs transplantation in clinical applications in the future.

\section{Conflicts of Interest}

The authors declare that there is no conflict of interest regarding the publication of this paper.

\section{Funding Statement}

This work was supported by grants from the National Nature Science Foundation of China (81470751 to Y.L, 81600891 to L.G, 81600829 to Y.S), the Beijing Natural Science Foundation (7172087 to Y.L), the Beijing Municipal Administration of Hospitals Clinical Medicine Development of Special Funding Support (ZYLX201703 to Y.B), the Beijing Baiqianwan Talents Project (2017A17 to Y.L), the Beijing Excellent Talent (2014000021469G251 to L.G), and the Capital Characteristic Clinic Project (Z161100000516203 to L.G).

\section{References}

1. Nazir MA (2017) Prevalence of periodontal disease, its association with systemic diseases and prevention. International Journal of Health Sciences 11: 72-80.

2. Van DT (2008) The management of inflammation in periodontal disease. Journal of Periodontology 79: 1601-1608.

3. D Graves (2008) Cytokines that promote periodontal tissue destruction. Journal Periodontology 79: 1585-1591.

4. Narayanan AS, Bartold PM (1996) Biochemistry of periodontal connective tissues and their regeneration: a current perspective. Connective Tissue Research 34: 191-201.

5. Zucchelli G, Bernardi F, Montebugnoli F (2002) Enamel matrix proteins and guided tissue regeneration with titanium-reinforced expanded polytetrafluoroethylene membranes in the treatment of infrabony defects: a comparative controlled clinical trial. Journal of Periodontology 73: 3-12.

6. Maeda V, Wada N, Tomokiyo A (2013) Prospective potency of TGF-beta1 on maintenance and regeneration of periodontal tissue. International Review of Cell and Molecular Biology 283-367.

7. Tsesis I, Rosen E, Tamse A (2011) Effect of guided tissue regeneration on the outcome of surgical endodontic treatment: a systematic review and meta-analysis. Journal of Endodontics 37: 1039-1045.

8. Feng J, Mantesso A, Bari DC (2011) Dual origin of mesenchymal stem cells contributing to organ growth and repair. Proceedings of the national Academy of Science 108: 6503-6508.

9. Teitelbaum SL (2010) Stem cells and osteoporosis therapy. Cell Stem Cell 7: 553-554.

10. Seo BM, Miura M, Gronthos S (2004) Investigation of multipotent postnatal stem cells from human periodontal ligament. Lancet 364 : 149-155.

11. Liu Y, Zheng Y, Ding G (2008) Periodontal ligament stem cell-mediated treatment for periodontitis in miniature swine. Stem Cells 26: 1065-1073.

12. Ding G, Liu Y, Wang W (2010) Allogeneic periodontal ligament stem cell therapy for periodontitis in swine. Stem Cells 28: 1829-1838.

13. Liu O, Xu J, Ding G (2013) Periodontal ligament stem cells regulate B lymphocyte function via programmed cell death protein 1 . Stem Cells 31 : 1371-1382.

14. Doğan A, Ozdemir A, Kubar A (2002) Assessment of periodontal healing by seeding of fibroblast-like cells derived from regenerated periodontal ligament in artificial furcation defects in a dog: a pilot study. Tissue Engineering 8: 273-282.

15. Doğan A, Ozdemir A, Kubar A (2003) Healing of artificial fenestration defects by seeding of fibroblast-like cells derived from regenerated periodontal ligament in a dog: a preliminary study. Tissue Engineering 9: 1189-1196.

16. Nakahara T, Nakamura T, Kobayashi E (2004) In situ tissue engineering of periodontal tissues by seeding with periodontal ligament-derived cells. Tissue Engineering 10: 537-544.

17. Bruckmann C, Walboomers V, Matsuzaka K (2005) Periodontal ligament and gingival fibroblast adhesion to dentin-like textured surfaces. Biomaterials 26: 339-346.

18. Yamada Y, Ueda M, Hibi H (2006) A novel approach to periodontal tissue regeneration with mesenchymal stem cells and platelet-rich plasma using tissue engineering technology: a clinical case report. The International Journal of Periodontics \& Restorative Dentistry 26: 363-369.

19. Yang Y, Rossi FM, Putnins EE (2010) Periodontal regeneration using engineered bone marrow mesenchymal stromal cells. Biomaterials 31 : 8574-8582.

20. Yang ZH, Zhang XJ, Dang NN (2009) Apical tooth germ cell-conditioned medium enhances the differentiation of periodontal ligament stem cells into cementum/periodontal ligament-like tissues. Journal of Periodontal Research 44: 199-210.

21. Park CH, Rios HF, Jin Q (2010) Biomimetic hybrid scaffolds for engineering human tooth-ligament interfaces. Biomaterials 31 : 5945-5952. 
Citation: Han N, Su Y, Guo L, Jia L, Du J, et al. (2018) The Clinical Effect and Meta-analysis of Mesenchymal Stem Cells for Periodontal Tissue Regeneration. Dentistry 8: 508. doi:10.4172/2161-1122.1000508

Page 6 of 6

22. Washio K, Iwata T, Mizutani M (2010) Assessment of cell sheets derived from human periodontal ligament cells: a pre-clinical study. Cell and Tissue Research 341: 397-404.

23. Tsumanuma Y, Iwata T, Washio K (2011) Comparison of different tissuederived stem cell sheets for periodontal regeneration in a canine 1-wall defect model. Biomaterials 32: 5819-5825.

24. Feng F, Akiyama K, Liu Y (2010) Utility of PDL progenitors for in vivo tissue regeneration: a report of 3 cases. Oral Diseases 16: 20-28.

25. Baba S, Yamada Y, Komuro A (2016) Phase I/II trial of autologous bone marrow stem cell transplantation with a three-dimensional woven-fabric scaffold for periodontitis. Stem Cells International 2016: 1-7.

26. Aquino R, Rosa DA, Lanza V (2009) Human mandible bone defect repair by the grafting of dental pulp stem/progenitor cells and collagen sponge biocomplexes. European Cells \& Materials 18: 75-83.

27. Dhote R, Charde P, Bhongade $M$ (2015) Stem cells cultured on beta tricalcium phosphate $(\beta-\mathrm{TCP})$ in combination with recombinant human platelet-derived growth factor- BB (rh-PDGF-BB) for the treatment of human infrabony defects. Journal of Stem Cells 10: 243-254.

28. Chen FM, Gao LN, Tian BM (2016) Treatment of periodontal intrabony defects using autologous periodontal ligament stem cells: a randomized clinical trial. Stem Cell Research \& Therapy 7: 33.
29. Zanwar K, Kumar GK, Bhongade ML (2017) Efficacy of human umbilical stem cells cultured on polylactic/ polyglycolic acid membrane in the treatment of multiple gingival recession defects: a randomized controlled clinical study. Journal of Dentistry 18: 95-103.

30. Tassi SA, Sergio NZ, Misawa MYO (2017) Efficacy of stem cells on periodontal regeneration: Systematic review of pre-clinical studies. Journal of Periodontal Research 52: 793-812.

31. Yan XZ, Yang F, Jansen JA (2015) Cell-based approaches in periodontal regeneration: a systematic review and meta-analysis of periodontal defect models in animal experimental work. Tissue Engineering. Part B. Review 21: 411-426.

32. Dangaria SJ, Ito Y, Luan X (2011) Successful periodontal ligament regeneration by periodontal progenitor preseeding on natural tooth root surfaces. Stem Cells and Development 20: 1659-1668.

33. Bright R, Hynes K, Gronthos S (2014) Periodontal ligament-derived cells for periodontal regeneration in animal models: a systematic review. Journal of Periodontal Research 50: 160-172. 